\title{
Overexpression and clinical significance of IBP in epithelial ovarian carcinoma
}

\author{
YINGJUAN XU ${ }^{1}$, YANGMING HOU ${ }^{2}$, TIANBO LIU ${ }^{1}$ and GE LOU ${ }^{1}$ \\ ${ }^{1}$ Department of Gynecology, The Affiliated Tumor Hospital, Harbin Medical University, Harbin, Heilongjiang 150081; \\ ${ }^{2}$ Department of Hepatobiliary and Pancreatic Surgery, Jilin Cancer Hospital, Changchun, Jilin 130012, P.R. China
}

Received November 1, 2016; Accepted November 16, 2017

DOI: $10.3892 / \mathrm{ol} .2018 .8125$

\begin{abstract}
Interferon regulatory factor-4 binding protein (IBP) is as a type of $\rho$ GTPase suggested to serve an important role in tumor occurrence and development through the effects of cytoskeletal remodeling, and cell conduction mechanism. IBP is widely expressed in the immune system and expressed in several types of tumors. However, its expression and prognostic value in epithelial ovarian carcinoma (EOC) remain unclear. The present study aimed to investigate the expression of IBP in EOC, and its effect on clinicopathological variables and prognosis. A total of 107 and 30 cases of epithelial ovarian carcinoma and benign ovarian disease tissue sections, respectively, were examined using immunohistochemistry. The results indicated that the IBP expression status was negative or markedly weak in normal tissues, but highly expressed in EOC tissues. A significant association was observed between IBP overexpression and various clinicopathological factors, including advanced International Federation of Gynecology and Obstetrics stage $(\mathrm{P}<0.001)$, poor histologic grade $(\mathrm{P}=0.002)$, peritoneal carcinomatosis $(\mathrm{P}<0.001)$, lymph-node metastasis $(\mathrm{P}=0.023)$ and recurrence $(\mathrm{P}<0.001)$. Multivariate Cox regression analysis additionally suggested that IBP overexpression was an independent factor affecting recurrence-free survival [hazard ratio $(\mathrm{HR})=4.099 ; 95 \%$ confidence interval (CI), 2.209-7.606; $\mathrm{P}<0.001)$ and overall survival $(\mathrm{HR}=2.317$; $95 \%$ CI, 1.484-3.617; $\mathrm{P}<0.001)$ in patients with EOC. The results of the present study demonstrated that IBP overexpression may be associated with tumor development and progression in EOC, and therefore may serve as a novel target for treating this disease.
\end{abstract}

\section{Introduction}

Ovarian carcinoma is one of the most common malignancies of the female reproductive system and remains a major global

Correspondence to: Professor Ge Lou, Department of Gynecology, The Affiliated Tumor Hospital, Harbin Medical University, 6 Baojian Road, Nangang, Harbin, Heilongjiang 150081, P.R. China

E-mail: dr_lougehmu@163.com

Key words: epithelial ovarian carcinoma, interferon regulatory factor-4 binding protein, immunohistochemistry, prognosis, survival health problem. In 2012, 238,700 new cases were diagnosed, and 151,900 females succumbed to this disease worldwide (1). One of the most common types of ovarian cancer is epithelial ovarian cancer (EOC), which accounts for $\sim 90 \%$ of all cases (2). Due to the lack of early diagnostic methods EOC is usually diagnosed at a late stage, reducing the chance of successful treatment (3). The primary factor that limits the efficacy of treatment in EOC is chemotherapy resistance. In previous years, there have been advances in medical treatments and several novel chemotherapeutic regimens have been identified in EOC; however, these continue to result in poor prognoses. Therefore, there is a requirement to characterize the pathogenesis of EOC and identify novel and efficient prognostic markers.

Interferon regulatory factor-4 binding protein (IBP), which had been isolated from the human cDNA library, was first identified during the search for the potential partner of the lymphoid-restricted transcription factor interferon regulatory (IRF-4) in 2003 (4). IBP is primarily expressed in lymphocytes and located in the cytoplasm. As IBP is a type of protein that participates in the formation of $\mathrm{T}$ cell-mediated immunity synapses under T-cell receptor signal stimulation and associates with the activity of T-helper cell (Th)2 cells, early studies of IBP focused on the lymphatic system $(4,5)$. Through the inhibition of IRF-4 transcription factor activity, IBP may also decrease the generation of interleukin-17/21, and affect the function of Th17 cells $(6,7)$. In addition, IBP participates in the regulation of the Toll-like receptor signal transduction pathway, in which mitogen-activated protein kinase and nuclear factor- $\kappa \mathrm{B}$ are involved. Therefore, IBP serves an important function in maintaining immune homeostasis. IBP-knockout mice exhibited symptoms of autoimmune disease (8). Gene expression profiles demonstrate that IBP is one of the top five differentially expressed genes in extraskeletal myxoid chondrosarcoma (9). IBP may also be considered as a guanine nucleotide exchange factor (GEF), which activates the $\rho$ GTPase family members, including ras-related $\mathrm{C} 3$ botulinum toxin substrate 1 (Rac1), cell division control protein 42 homolog and ras homolog gene family, member A (10). The $\rho$ GTPase family serves an important function in the metabolism of tumor cells, and the progression of tumor proliferation, migration and invasion (11). In addition, the high expression of GEF and the guanosine triphosphate (GTP) $\rho$ enzyme family are often associated with tumor occurrence (11). Therefore, IBP molecules may have a direct or indirect effect on cytoskeleton remodeling, and cell 
transmission mechanism, which serves a certain function in tumor development. Consequently, IBP may serve as a novel and efficient prognostic marker. At present, previous studies investigating IBP and its effects in tumors are limited. Existing data suggests that IBP may promote the development of breast cancer and colorectal cancer, and increases the invasiveness of oral squamous cell carcinoma (12-14). However, the expression and prognostic value of IBP in ovarian cancer remain unknown.

The present study aimed to reveal the expression of IBP in epithelial ovarian cancer and its association with clinicopathological features, then determine whether IBP may perform as a novel prognostic factor of EOC.

\section{Materials and methods}

Patients and tissue samples. All the paraffin-embedded samples and clinical data were selected from the Department of Gynecologic Oncology, Third Affiliated Hospital of Harbin Medical University (Harbin, China) between January 2008 and January 2011. The inclusion criteria of ovarian cancer patients were as follows: i) Diagnosed with ovarian cancer via pathology; ii) did not suffer from other malignant diseases; and iii) patients had not undergone any preoperative treatment, including chemotherapy or radiotherapy. Exclusion criteria were listed as: i) Was diagnosed with serious systemic disease; ii) the tumor had distant metastasis; iii) the patient had not received a radical resection. A total of 107 patients who met the aforementioned criteria were enrolled in the present study aged 22-76 years old (with a median age of 54 years old). All patients with ovarian cancer were treated with cytoreductive surgery, which was followed by six courses of standard platinum-based combination chemotherapy, consisting of six courses of treatment with three weeks between each course.

The surgical staging of all patients was confirmed based on The International Federation of Gynecology and Obstetrics stage (FIGO) and subsequently classified into early (I-II) and advanced stages (III-IV) (15). Histological type and tumor grade were based on the World Health Organization classification standard (16). Histological grade (tumor differentiation) was determined in accordance with the standard Silverberg grading system (17), and classified into low (G1) and high levels (G2-G3). A complete set of all parameters was obtained; these parameters included patient age, histological grade, clinical stage, CA-125 level, ascites volume, lymph-node metastasis and peritoneal carcinomatosis, and whether recurrence, overall survival (OS), and recurrence-free survival (RFS) occurred. The study protocol was approved by the Ethics Committee of the Third Affiliated Hospital of Harbin Medical University and all patients provided written informed consent.

For survival analysis, all enrolled patients with ovarian cancer were followed up periodically until mortality or up to 5 years following surgery. The median follow-up time was 48 months (ranging from 3-60 months). Within 2 years following surgery, examinations, including serum cancer antigen (CA)-125, pelvic magnetic resonance imaging, chest X-ray and color Doppler ultrasound of abdomen, were performed every 3 months. During the postoperative period of 3-5 years, the aforementioned tests were repeated once every 6 months and annually thereafter. RFS was defined as the time span between the date of surgery and diagnosed recurrence or distant metastasis. OS was measured as the time interval from the date of surgery to mortality, which included all-cause mortality or the termination of the follow-up period of the present study.

Immunohistochemical (IHC) staining. Slices (4- $\mu$ m thick) were cut from paraffin-embedded specimens stored by the pathology department of The Third Affiliated Hospital of Harbin Medical University (Harbin, China), and hematoxylin and eosin (HE) staining was conducted on these samples. IHC staining was performed on tissue slices adjacent to the HE-stained section using an avidin-biotin immunoperoxidase technique, as follows. These sections were incubated at $80^{\circ} \mathrm{C}$ for $30 \mathrm{~min}$, deparaffinized with xylene, and rehydrated with different alcohol concentrations (Analytically pure ethanol concentrations were: 100, 95 and $80 \%$, each for 5-10 min). All dewaxed slices were immersed in $0.01 \mathrm{mmol} / 1$ citrate buffer $(\mathrm{pH} \mathrm{6.0)}$ and maintained in high-pressure steam at $121^{\circ} \mathrm{C}$ for 4 min to fix antigenicity. Subsequently, the slices were cooled to room temperature. To remove the endogenous peroxidase activity, the slices were placed in $3 \%$ hydrogen peroxide for $10 \mathrm{~min}$ at room temperature. Tissue sections were incubated with IBP-specific mouse monoclonal antibodies (dilution 1:100; Abcam, Cambridge, UK; cat. no. ab57228) at $4^{\circ} \mathrm{C}$ overnight, washed three times ( 5 min each) with PBS, and finally incubated at $37^{\circ} \mathrm{C}$ with biotin-labeled secondary antibody (goat anti-mouse immunoglobulin G; undiluted; cat. no. Pv6000; OriGene Technologies, Inc., Rockville, MD, USA) and horseradish peroxidase-conjugated streptavidin for $20 \mathrm{~min}$. All slices were immersed in 3,3 diaminobenzidine tetrahydrochloride (Dako; Agilent Technologies, Inc., Santa Clara, CA, USA) at $37^{\circ} \mathrm{C}$, which was followed by Meyer hematoxylin (undiluted) at room temperature for $1 \mathrm{~min}$ to counterstain. The negative control sections were incubated at $4^{\circ} \mathrm{C}$ overnight with rabbit serum (undiluted; cat. no. AR0010; Boster Biological Technology Co., Ltd., Wuhan, China), instead of primary antibodies.

Evaluation of IBP expression by IHC. All slices were independently assessed by two experienced pathologists who were blind to the clinical pathology results and other patient information. IBP expression level was evaluated via the proportion of positively stained cells and intensity of tumor cells. The semi-quantitative evaluation criteria were as follows: The score of all sections was based upon the intensities and proportions of IBP staining in the tumor cells. The percentage of positive tumor cells was scored according to the following: 1, 0-10\% of positive cells; $2,11-50 \%$ of positive cells; $3,51-80 \%$ of positive cells; and $4,>80 \%$ of the positive cells. The IBP protein expression levels were scored as follows: 0 , unstained; 1 , weak positive staining; 2 , moderate staining; and 3, marked staining. The final score was calculated by multiplying the percentage score with the expression level score $(0,1,2,3,4,6,8,9$ or 12$)$. Scores of 0 were categorized as negative IBP staining, scores between 1-4 were considered weak positives for IBP staining, and scores $>4$ were classified as strong positives for IBP staining. Slices with inconsistent results were re-examined by the original two pathologists and a senior pathologist until a consensus was reached.

Statistical analysis. All data are presented as the mean \pm the standard deviation. A $\chi^{2}$ test was used to analyze the association between IBP overexpression and clinicopathological variables. OS and RFS were calculated according to the Kaplan-Meier 

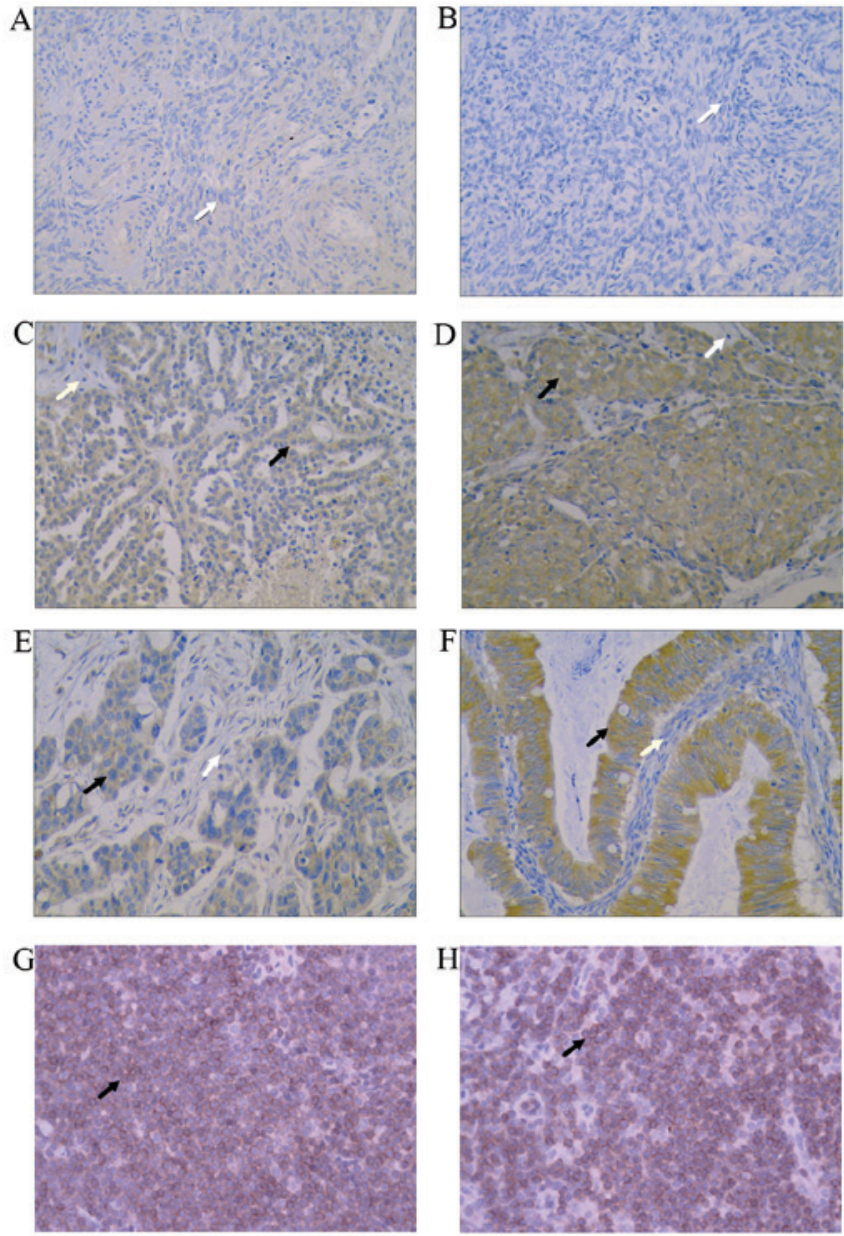

Figure 1. Representative immunohistochemical staining for IBP expression in ovarian specimens. (A) Negative expression of IBP in normal ovarian tissue. (B) Negative control in ovarian carcinoma. (C, D) Low expression of IBP in epithelial ovarian carcinoma: (C) IBP expression in low-level serous epithelial ovarian carcinoma; (D) IBP expression in high-level serous epithelial ovarian carcinoma. The positive cells were stained brown and diffusely distributed. (E, F) High expression of IBP in epithelial ovarian carcinoma: (E) IBP expression in high-level serous epithelial ovarian carcinoma; (F) IBP expression in endometrioid epithelial ovarian carcinoma. $(\mathrm{G}, \mathrm{H})$ Positive expression of IBP in lymphoid tissue. The white arrows indicate negative stained cells, and the black arrows indicate positive stained cells. Original magnification, $\mathrm{x} 400$. IBP, interferon regulatory factor-4 binding protein.

method, and a univariate analysis of the log-rank test was used to evaluate the differences among the levels of potential prognostic factors. In the multivariate analysis, a Cox proportional hazard regression model was used to assess the independent predictive factors of OS and RFS. $\mathrm{P}<0.05$ was considered to indicate a statistically significant difference. All statistical analyses were performed using SPSS version 19.0 software (IBM Corp., Armonk, NY, USA).

\section{Results}

IBP is highly expressed in patients with epithelial ovarian carcinoma. Representative immunostaining of IBP is demonstrated in Fig. 1. The positive cells were stained brown. Fig. 1A indicates the negative expression of IBP in normal ovarian tissue and Fig. 1B exhibits the negative control in ovarian carcinoma. Fig. $1 \mathrm{C}$ and $\mathrm{D}$
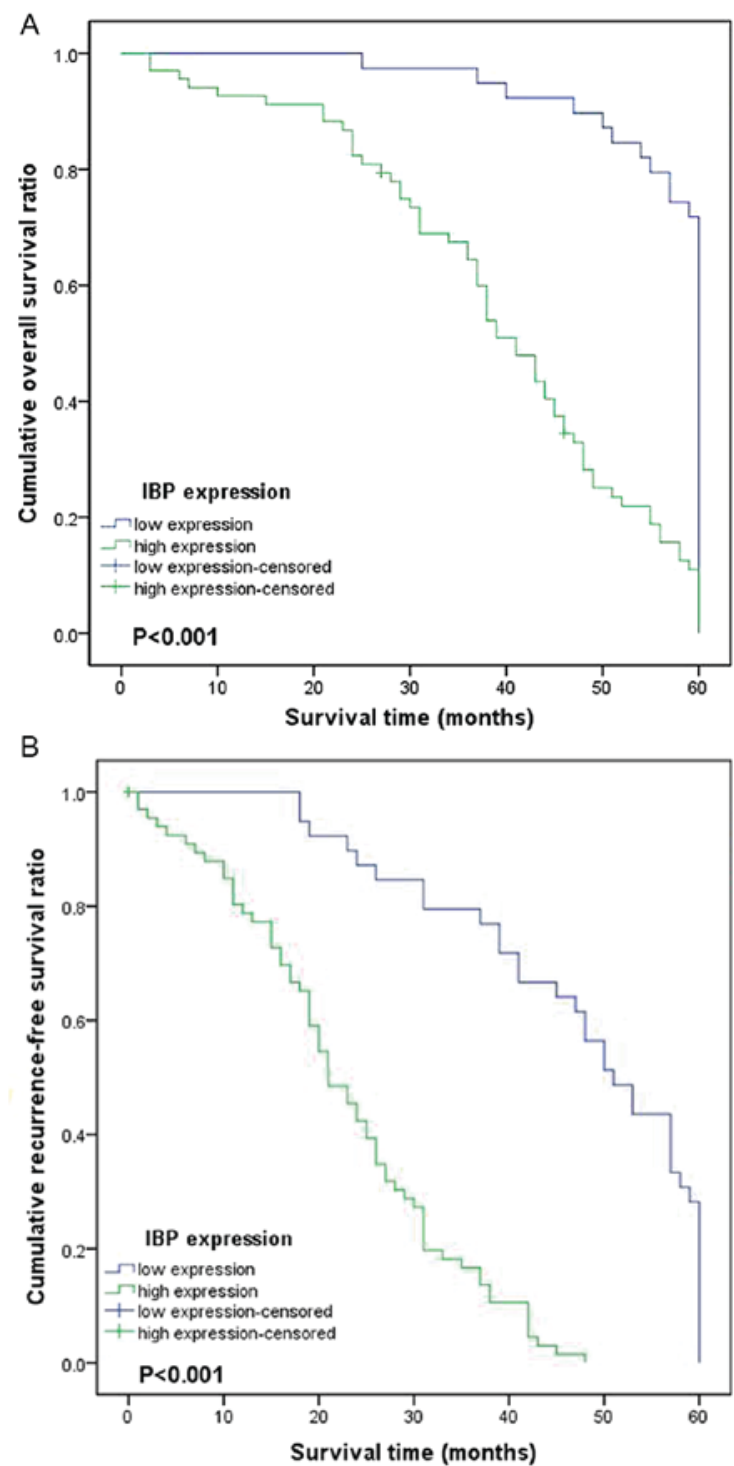

Figure 2. Kaplan-Meier analysis for the association between IBP expression and survival. The (A) overall and the (B) recurrence-free survival of patients with ovarian carcinoma with high, and low expression are presented. The log-rank test demonstrated that patients with low IBP staining exhibited a significantly improved overall and recurrence-free survival vs. patients with high expression. IBP, interferon regulatory factor-4 binding protein.

demonstrate a low expression of IBP in low-level and high-level serous epithelial ovarian carcinoma, respectively. Fig. $1 \mathrm{E}$ and $\mathrm{F}$ indicate a strong positive expression of IBP in high-level serous epithelial ovarian carcinoma and endometrioid epithelial ovarian carcinoma. Fig. $1 \mathrm{G}$ and $\mathrm{H}$ demonstrate positive expression of IBP in lymphoid tissue. In the present study, IBP was not expressed in normal ovary tissues (Fig. 1A). However, in the epithelial ovarian carcinoma tissue samples, 39/107 (36.4\%) slices exhibited low IBP expression, and 68/107 (63.6\%) sections indicated high IBP expression. In epithelial ovarian carcinoma tissue samples, as indicated in Fig. 1, IBP staining appeared as brown particles.

Association between IBP protein expression and clinicopathological parameters. Table I summarizes the associations between the expression of IBP in ovarian cancer and clinicopathological variables. High IBP overexpression was associated with high FIGO 
Table I. Association between interferon regulatory factor-4 binding protein overexpression and clinicopathological characteristics of ovarian carcinoma.

\begin{tabular}{|c|c|c|c|c|}
\hline \multirow[b]{2}{*}{ Variable } & \multirow[b]{2}{*}{ No. } & \multicolumn{2}{|c|}{ IBP expression } & \multirow[b]{2}{*}{ P-value } \\
\hline & & Low, n (\%) & High, n (\%) & \\
\hline \multicolumn{5}{|l|}{ Age, years } \\
\hline$\leq 50$ & 34 & $16(47.1)$ & $18(52.9)$ & \\
\hline$>50$ & 73 & $23(31.5)$ & $50(68.5)$ & 0.120 \\
\hline \multicolumn{5}{|l|}{ Histological type } \\
\hline Serous & 62 & $23(37.1)$ & $39(62.9)$ & \\
\hline Mucinous & 19 & $5(26.3)$ & $14(73.7)$ & \\
\hline Endometrioid & 21 & $11(52.4)$ & $10(47.6)$ & \\
\hline Clear cell & 5 & $0(0.0)$ & $5(100.0)$ & 0.054 \\
\hline \multicolumn{5}{|c|}{ Histological grade } \\
\hline G1 & 16 & $11(68.8)$ & $5(31.2)$ & \\
\hline G2/G3 & 91 & $26(28.6)$ & $65(71.4)$ & 0.002 \\
\hline \multicolumn{5}{|l|}{ FIGO stage } \\
\hline $\mathrm{I}+\mathrm{II}$ & 23 & $21(91.3)$ & $2(8.7)$ & \\
\hline $\mathrm{III}+\mathrm{IV}$ & 84 & $18(21.4)$ & $66(78.6)$ & $<0.001$ \\
\hline \multicolumn{5}{|c|}{ Serum CA-125 level, U/ml } \\
\hline$\leq 35$ & 12 & $6(50.0)$ & $6(50.0)$ & \\
\hline$>35$ & 95 & $33(34.7)$ & $62(65.3)$ & 0.301 \\
\hline \multicolumn{5}{|c|}{ Lymph node metastasis } \\
\hline Yes & 62 & $17(28.8)$ & $42(71.2)$ & \\
\hline No & 45 & $22(45.8)$ & $26(54.2)$ & 0.023 \\
\hline \multicolumn{5}{|c|}{ Peritoneal metastasis } \\
\hline Yes & 72 & $18(25.0)$ & $54(75.0)$ & \\
\hline No & 35 & $21(60.0)$ & $14(40.0)$ & $<0.001$ \\
\hline \multicolumn{5}{|l|}{ Ascites, $\mathrm{ml}$} \\
\hline$<100$ & 43 & $17(39.5)$ & $26(60.5)$ & \\
\hline$\geq 100$ & 64 & $22(34.4)$ & $42(65.6)$ & 0.587 \\
\hline \multicolumn{5}{|l|}{ Recurrence } \\
\hline Yes & 26 & $20(76.9)$ & $6(23.1)$ & \\
\hline No & 81 & $19(23.5)$ & $62(76.5)$ & $<0.001$ \\
\hline
\end{tabular}

${ }^{a} \chi^{2}$ test. FIGO, International Federation of Gynecology and Obstetrics G1, well-differentiated; G2, moderately differentiated; G3, poorly differentiated; CA-125, cancer antigen-125.

stage $(\mathrm{P}<0.001)$, histologic grade $(\mathrm{P}=0.002)$, relapse rate $(\mathrm{P}<0.001)$, lymphatic metastasis $(\mathrm{P}=0.023)$, and peritoneal carcinomatosis $(\mathrm{P}<0.001)$.Incontrast,nostatistically significantassociationsbetween IBP and age $(\mathrm{P}=0.120)$, histological type $(\mathrm{P}=0.054)$, preoperative serum $\mathrm{CA}-125(\mathrm{P}=0.301)$, ascites $(\mathrm{P}=0.587)$ or other pathological parameters were observed.

To investigate the potential clinical utility of IBP overexpression, the association between IBP and OS, and RFS outcomes in 107 cases of epithelial ovarian carcinoma specimens was assessed. Kaplan-Meier and log-rank test methods were used, and it was identified that a high expression of IBP was associated with relatively short RFS and OS times compared with the low expression group $(\mathrm{P}<0.001$ and $\mathrm{P}<0.001$, respectively; Table II; Fig. 2). In addition, alongside IBP overexpression, FIGO stage, histological type, histological grade, lymph node metastasis and peritoneal metastasis were associated with epithelial ovarian cancer prognosis.

Parameters identified as significant in the univariate Kaplan-Meier analysis were included in the multivariate Cox regression model. The multivariate Cox regression analysis indicated that IBP may serve as an independent prognostic marker for OS [hazard ratio $(\mathrm{HR})=2.317 ; 95 \%$ confidence interval $(\mathrm{CI}), 1.484-3.617 ; \mathrm{P}<0.001]$ and $\mathrm{RFS}(\mathrm{HR}=4.099 ; 95 \%$ CI, 2.209-7.606; $\mathrm{P}<0.001)$ in EOC (Table III). Furthermore, FIGO stage $(\mathrm{P}=0.029$ and $\mathrm{P}=0.002$, respectively), lymph node $(\mathrm{P}=0.022$ and $\mathrm{P}=0.013$, respectively), and peritoneal metastasis $(\mathrm{P}=0.014$ and $\mathrm{P}=0.020$, respectively) were significantly associated with OS and RFS. However, histological grade and histological type were not independent predictive factors for RFS and OS. 
Table II. Univariate survival analysis of OS and RFS in 107 patients with ovarian carcinoma.

\begin{tabular}{|c|c|c|c|c|c|c|c|}
\hline \multirow[b]{2}{*}{ Variable } & \multirow{2}{*}{$\begin{array}{c}\text { No. } \\
(n=107)\end{array}$} & \multicolumn{2}{|c|}{ OS, months } & \multirow[b]{2}{*}{ P-value ${ }^{a}$} & \multicolumn{2}{|c|}{ RFS, months } & \multirow[b]{2}{*}{ P-value ${ }^{a}$} \\
\hline & & Mean \pm SE & $95 \% \mathrm{CI}$ & & Mean $\pm \mathrm{SE}$ & $95 \% \mathrm{CI}$ & \\
\hline Age, years & & & & 0.145 & & & 0.395 \\
\hline$\leq 50$ & 34 & $48.029 \pm 2.581$ & $42.970-53.089$ & & $34.353 \pm 3.061$ & $28.353-40.353$ & \\
\hline$>50$ & 73 & $44.715 \pm 1.827$ & 41.133-48.296 & & $30.535 \pm 2.014$ & 26.589-34.482 & \\
\hline Histological type & & & & $<0.001$ & & & 0.005 \\
\hline Serous & 62 & $45.450 \pm 1.956$ & $41.617-49.283$ & & $30.967 \pm 2.157$ & 26.739-35.196 & \\
\hline Mucinous & 19 & $48.105 \pm 3.589$ & $41.070-55.140$ & & $33.474 \pm 4.239$ & $25.164-41.783$ & \\
\hline Endometrioid & 21 & $49.125 \pm 3.145$ & $42.961-55.289$ & & $36.700 \pm 3.844$ & $29.165-44.235$ & \\
\hline Clear cell & 5 & $27.000 \pm 2.811$ & 21.491-32.509 & & $15.400 \pm 3.750$ & $8.051-22.749$ & \\
\hline Histological grade & & & & 0.002 & & & 0.003 \\
\hline G1 & 16 & $57.188 \pm 1.239$ & $54.759-59.616$ & & $46.688 \pm 3.454$ & $39.917-53.458$ & \\
\hline G2/G3 & 91 & $43.761 \pm 1.656$ & $40.514-47.007$ & & $29.090 \pm 1.748$ & $25.663-32.516$ & \\
\hline FIGO stage & & & & $<0.001$ & & & $<0.001$ \\
\hline $\mathrm{I}+\mathrm{II}$ & 23 & $59.000 \pm 1.000$ & $57.040-60.960$ & & $53.261 \pm 1.951$ & $49.437-57.085$ & \\
\hline III+IV & 84 & $42.108 \pm 1.676$ & $38.823-45.392$ & & $25.744 \pm 1.526$ & $22.752-28.735$ & \\
\hline Serum CA-125 level, U/ml & & & & 0.451 & & & 0.519 \\
\hline$\leq 35$ & 12 & $49.667 \pm 3.722$ & $42.371-56.962$ & & $34.417 \pm 5.391$ & $23.849-44.984$ & \\
\hline$>35$ & 95 & $45.289 \pm 1.614$ & $42.125-48.453$ & & $31.430 \pm 1.779$ & 27.944-34.916 & \\
\hline Lymph node metastasis & & & & 0.001 & & & 0.001 \\
\hline Yes & 62 & $41.455 \pm 2.060$ & $37.148-45.492$ & & $26.672 \pm 2.103$ & $22.551-30.793$ & \\
\hline No & 45 & $51.696 \pm 1.814$ & $48.141-55.251$ & & $38.841 \pm 2.415$ & $34.108-43.574$ & \\
\hline Peritoneal metastasis & & & & $<0.001$ & & & $<0.001$ \\
\hline Yes & 72 & $41.376 \pm 1.861$ & $37.729-45.023$ & & $25.757 \pm 1.705$ & 22.415-29.099 & \\
\hline No & 35 & $54.714 \pm 1.685$ & $51.411-58.017$ & & $43.800 \pm 2.805$ & $38.303-49.297$ & \\
\hline Ascites, ml & & & & 0.102 & & & 0.070 \\
\hline$<100$ & 43 & $48.958 \pm 2.055$ & $44.931-52.986$ & & $35.341 \pm 2.752$ & $29.948-40.735$ & \\
\hline$\geq 100$ & 64 & $43.672 \pm 2.050$ & $39.654-47.690$ & & $29.484 \pm 2.097$ & $25.375-33.594$ & \\
\hline IBP expression & & & & $<0.001$ & & & $<0.001$ \\
\hline Low & 39 & $56.718 \pm 1.208$ & $54.350-59.086$ & & $47.103 \pm 2.244$ & $42.705-51.500$ & \\
\hline High & 68 & $39.401 \pm 1.852$ & $35.771-43.031$ & & $22.712 \pm 1.445$ & $19.880-25.544$ & \\
\hline
\end{tabular}

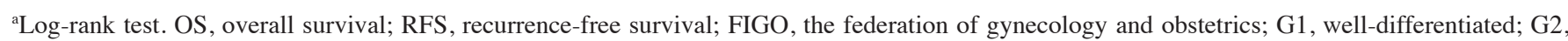
moderately differentiated; G3, poorly differentiated; SE, standard error; CI, confidence interval; CA-125, cancer antigen-125.

\section{Discussion}

To the best of our knowledge, the present study was the first to reveal the expression of IBP in primary untreated epithelial ovarian carcinoma and normal ovarian tissue through IHC using paraffin-embedded samples. In addition, the present study investigated the association of IBP overexpression with clinicopathological factors and prognosis of patients with ovarian cancer. Analysis on the experimental data of 107 patients with ovarian cancer indicated that high IBP expression was associated with tumor recurrence, metastasis and a shorter OS or RFS time These data indicate that IBP may be an independent prognostic factor for epithelial ovarian carcinoma.

In previous years, the expression of IBP has been explored: Several studies have identified that IBP was overexpressed, and served an important role in several types of carcinomas, including breast cancer $(13,18)$, colorectal cancer $(12)$ and oral squamous cell carcinoma (OSCC) (14). However, prior to the present study, the expression and prognostic value of IBP in epithelial ovarian carcinoma was unclear. In the present study, the association between IBP expression and clinicopathological features in EOC was analyzed. It was observed that IBP was markedly expressed in EOC specimens, but not in normal ovarian tissue. High IBP expression was significantly associated with high FIGO stage, poor differentiation, high relapse rate, lymphatic metastasis, histological type and peritoneal carcinomatosis. In addition, the Kaplan-Meier method and log-rank test data also suggested that the patients with high IBP expression exhibited significantly poor OS, and RFS in comparison with patients with low expression of IBP. The Cox proportional 
Table III. Multivariate Cox regression analysis for various potential prognostic characteristics of OS and RFS in 107 patients with ovarian carcinoma.

\begin{tabular}{|c|c|c|c|c|c|c|}
\hline \multirow[b]{2}{*}{ Variable } & \multicolumn{3}{|c|}{ OS } & \multicolumn{3}{|c|}{ RFS } \\
\hline & $\operatorname{Exp}(B)$ & $95 \% \mathrm{CI}$ & P-value ${ }^{a}$ & $\operatorname{Exp}(B)$ & $95 \% \mathrm{CI}$ & P-value ${ }^{a}$ \\
\hline FIGO stage & 1.803 & $1.062-3.059$ & 0.029 & 2.623 & $1.426-4.823$ & 0.002 \\
\hline Histological type & 1.119 & $0.886-1.412$ & 0.337 & 1.120 & $0.891-1.407$ & 0.332 \\
\hline Histological grade & 1.240 & $0.701-2.193$ & 0.460 & 1.651 & $0.942-2.893$ & 0.080 \\
\hline Lymph node metastasis & 1.587 & $1.067-2.359$ & 0.022 & 1.679 & $1.114-2.530$ & 0.013 \\
\hline Peritoneal metastasis & 1.725 & $1.118-2.660$ & 0.014 & 1.732 & $1.089-2.755$ & 0.020 \\
\hline IBP & 2.317 & $1.484-3.617$ & $<0.001$ & 4.099 & $2.209-7.606$ & $<0.001$ \\
\hline
\end{tabular}

${ }^{\mathrm{a} C}$ Cox regression test. OS, overall survival; RFS, recurrence-free survival; FIGO, the federation of gynecology and obstetrics; IBP, interferon regulatory factor-4 binding protein; $\mathrm{CI}$, confidence interval; $\operatorname{Exp}(\mathrm{B})$, exponentiation of the B coefficient.

hazard regression model demonstrated that IBP was an independent prognostic factor for OS and RFS in patients with EOC. This data suggested that IBP serves an important role in the carcinogenesis and tumor progression of EOC, and therefore may be a promising prognostic marker. These results are in accordance with previous studies demonstrating the roles of IBP in tumor progression in breast cancer $(13,18)$, colorectal cancer (12) and oral squamous cell carcinoma (14), and suggest an association between high IBP expression and unfavorable biological behavior in EOC including poor histological grade, high FIGO stage, lymph node and peritoneal metastasis. All these results suggest that IBP serves an important biological role in carcinogenesis and tumor progression.

At present, certain indicators attempt to explain the mechanisms by which IBP promotes cancer development. Specifically, IBP and GEF family molecules have similar structures. Therefore, IBP may exhibit GEF-like characteristics. GEF is a member of the diffuse B-cell lymphoma protein family that promotes the GDP/GTP exchange reaction converting inactivated GDP- $\rho$ into GTP- $\rho$, and participating in the regulation of $\rho$ GTPase family (19). The $\rho$ GTPase family is involved in the regulation of a number of physiological process, including the motility and polarity of the cell, cell proliferation, formation of cytoskeleton, cell cycle and cross-membrane signal transfer (20-22). $\rho$ GTPase also serves an important function in the apoptosis, division, metabolism, proliferation, migration and invasion processes of tumor cells by regulating the gene transcriptional activity (23). In addition, it may regulate the interaction between tumor cells and surrounding stromal cells $(23,24)$. Furthermore, the tumorigenic activity of $\rho$ GTPase family is reflected in its excessive expression in various types of tumors $(23,25,26)$. Saurin et al $(27)$ demonstrated that the $\rho$ GTPase family was involved in the migration, invasion and resistance of colon cancer. Zhang et al (18) suggested that D4-GDI, a type of $\rho$ GTPase family regulatory factor, may promote the invasion of breast cancer. In addition, further studies indicated that GEF and $\rho$ GTPase were expected to become a target for cancer treatment $(11,24)$.

IBP has a similar structure and function to members of the GEF family, and may participate in the activation and regulation of $\rho$ GTPase family molecules $(10,28)$. IBP may also work together with activated Rac1 to regulate cell morphology (29) and affect cell differentiation via its interaction with integrins (30). An intramolecular basic amino acid-rich region K328-R340 (KRREQREQRERRR) exists within the IBP molecule, thereby suggesting that this molecule may be transposed into the nucleus to regulate gene expression (31). These studies indicated that IBP has a significant role in tumorigenesis and development.

Jian et al (14) identified that IBP was ectopically expressed in certain cases of OSCC, and that its expression was significantly correlated with tumor size, clinical stage, differentiation and distant metastasis. In addition, the upregulation of IBP expression markedly promoted the proliferation of OSCC cells and its knockdown inhibited the proliferation of OSCC cells. They suggested that IBP shortened the G1 phase, and enhanced the proportion of tumor cells entering the $\mathrm{S}$ phase, potentially through increasing the expression of cyclin D1. Li et al (13) revealed that IBP was highly expressed in breast cancer, but expressed at low levels in normal breast tissues, and that the ectopic expression of IBP was correlated with the malignant behavior of human breast cancer cells. Furthermore, Chen et al (32) suggested that IBP-mediated suppression of autophagy promotes the growth and metastasis of breast cancer cells by activating the mechanistic target of rapamycin kinase complex 2/protein kinase B/forkhead box O3a signaling pathway. Yang et al (33) demonstrated that IBP was a target gene of tumor protein 53 and that it suppresses cisplatin-induced apoptosis of breast cancer cells. Zhang et al (12) also indicated that IBP was overexpressed in colorectal cancer, but not in normal tissues; such observations were associated with the occurrence and development of colorectal cancer.

In conclusion, the present study demonstrated that IBP was overexpressed in the majority of patients with ovarian carcinoma, and that increased IBP expression was significantly associated with advanced tumor aggressiveness and poor prognosis. Therefore, these data may be used as evidence for the additional study of IBP as a novel biomarker to predict the prognosis of epithelial ovarian carcinoma.

\section{Acknowledgements}

The present study was supported by the National Natural Science Foundation of China (grant no. 81472028). 


\section{References}

1. Torre LA, Bray F, Siegel RL, Ferlay J, Lortet-Tieulent J and Jemal A Global cancer statistics, 2012. CA Cancer J Clin 65: 87-108, 2015.

2. Katso RM, Manek S, Ganjavi H, Biddolph S, Charnock MF, Bradburn M, Wells M and Ganesan TS: Overexpression of H-Ryk in epithelial ovarian cancer: Prognostic significance of receptor expression. Clin Cancer Res 6: 3271-3281, 2000.

3. Kolwijck E, Lybol CJ, Bulten J, Vollebergh JH, Wevers RA and Massuger LF: Prevalence of cysts in epithelial ovarian cancer. Eur J Obstet Gynecol Reprod Biol 151: 96-100, 2010.

4. Gupta S, Fanzo JC, Hu C, Cox D, Jang SY, Lee AE, Greenberg S and Pernis AB: $T$ cell receptor engagement leads to the recruitment of IBP, a novel guanine nucleotide exchange factor, to the immunological synapse. J Biol Chem 278: 541-549, 2003.

5. Tanaka Y, Bi K, Kitamura R, Hong S, Altman Y, Matsumoto A, Tabata H, Lebedeva S, Bushway PJ and Altman A: SWAP-70-like adapter of T cells, an adapter protein that regulates early TCR-initiated signaling in Th2 lineage cells. Immunity 18: 403-414, 2003.

6. Canonigo-Balancio AJ, Fos C, Prod'homme T, Bécart S and Altman A: SLAT/Def6 plays a critical role in the development of Th17 cell-mediated experimental autoimmune encephalomyelitis. J Immunol 183: 7259-7267, 2009.

7. Chen Q, Gupta S and Pernis AB: Regulation of TLR4-mediated signaling by IBP/Def6, a novel activator of Rho GTPases. J Leukoc Biol 85: 539-543, 2009.

8. Fanzo JC, Yang W, Jang SY, Gupta S, Chen Q, Siddiq A, Greenberg S and Pernis AB: Loss of IRF-4-binding protein leads to the spontaneous development of systemic autoimmunity. J Clin Invest 116: 703-714, 2006.

9. Subramanian S, West RB, Marinelli RJ, Nielsen TO, Rubin BP, Goldblum JR, Patel RM, Zhu S, Montgomery K, Ng TL, et al: The gene expression profile of extraskeletal myxoid chondrosarcoma. J Pathol 206: 433-444, 2005.

10. Gupta S, Lee A, Hu C, Fanzo J, Goldberg I, Cattoretti G and Pernis AB: Molecular cloning of IBP, a SWAP-70 homologous GEF, which is highly expressed in the immune system. Hum Immunol 64: $389-401,2003$.

11. Lazer G and Katzav S: Guanine nucleotide exchange factors for RhoGTPases: Good therapeutic targets for cancer therapy? Cell Signal 23: 969-979, 2011.

12. Zhang Z, Wang Q, Li P, Zhou Y, Li S, Yi W, Chen A, Kong P and $\mathrm{Hu} \mathrm{C}$ : Overexpression of the interferon regulatory factor 4-binding protein in human colorectal cancer and its clinical significance. Cancer Epidemiol 33: 130-136, 2009.

13. Li P, Zhang Z, Wang Q, Li S, Zhang Y, Bian X, Chen A and Hu C: The ectopic expression of IFN regulatory factor 4-binding protein is correlated with the malignant behavior of human breast cancer cells. Int Immunopharmacol 9: 1002-1009, 2009.

14. Jian CX, Yang MZ, Li P, Xiong J, Zhang ZJ, Li CJ, Chen A, Hu CM, Zhou JX and Li SH: Ectopically expressed IBP promotes cell proliferation in oral squamous cell carcinoma. Cancer Invest 30: 748-756, 2012.
15. PecorelliS: Revised FIGO staging forcarcinoma of the vulva, cervix, and endometrium. Int J Gynaecol Obstet 105: 103-104, 2009.

16. Tavassoli FA and Devilee P: World health organization classification of tumor. Histopathology 30: 274-276, 2002.

17. Ioffe OB and Silverberg SG: A universal grading system for ovarian cancer. Contemporary Ob/gyn, 2000.

18. Zhang Y and Zhang B: D4-GDI, a Rho GTPase regulator, promotes breast cancer cell invasiveness. Cancer Res 66: 5592-5598, 2006.

19. Raimondi F, Felline A and Fanelli F: Catching functional modes and structural communication in Dbl family Rho guanine nucleotide exchange factors. J Chem Inf Model 55: 1878-1893, 2015.

20. Ridley AJ: Rho family proteins: Coordinating cell responses. Trends Cell Biol 11: 471-477, 2001

21. Bokoch GM: Regulation of cell function by Rho family GTPases. Immunol Res 21: 139-148, 2000.

22. Bar-Sagi D and Hall A: Ras and Rho GTPases: A family reunion. Cell 103: 227-238, 2000.

23. Vega FM and Ridley AJ: Rho GTPases in cancer cell biology. FEBS Lett 582: 2093-2101, 2008.

24. Karlsson R, Pedersen ED, Wang Z and Brakebusch C: Rho GTPase function in tumorigenesis. Biochim Biophys Acta 1796: 91-98, 2009.

25. Lin M and van Golen KL: Rho-regulatory proteins in breast cancer cell motility and invasion. Breast Cancer Res Treat 84: 49-60, 2004.

26. Banyard J, Anand-Apte B, Symons M and Zetter BR: Motility and invasion are differentially modulated by Rho family GTPases. Oncogene 19: 580-591, 2000

27. Saurin JC, Fallavier M, Sordat B, Gevrey JC, Chayvialle JA and Abello J: Bombesin stimulates invasion and migration of Isreco1 colon carcinoma cells in a Rho-dependent manner. Cancer Res 62: 4829-4835, 2002.

28. Mavrakis KJ, McKinlay KJ, Jones P and Sablitzky F: DEF6, a novel PH-DH-like domain protein, is an upstream activator of the Rho GTPases Rac1, Cdc42, and RhoA. Exp Cell Res 294: 335-344, 2004.

29. Oka T, Ihara S and Fukui Y: Cooperation of DEF6 with activated Rac in regulating cell morphology. J Biol Chem 282: 2011-2018, 2007.

30. Samson T, Will C, Knoblauch A, Sharek L, von der Mark K, Burridge $\mathrm{K}$ and Wixler V: Def-6, a guanine nucleotide exchange factor for Rac1, interacts with the skeletal muscle integrin chain alpha7A and influences myoblast differentiation. J Biol Chem 282: 15730-15742, 2007.

31. Christophe D, Christophe-Hobertus C and Pichon B: Nuclear targeting of proteins. How many different signals? Cell Signal 12: 337-341, 2000

32. Chen S, Han Q, Wang X, Yang M, Zhang Z, Li P, Chen A, Hu C and Li S: IBP-mediated suppression of autophagy promotes growth and metastasis of breast cancer cells via activating mTORC2/Akt/FOXO3a signaling pathway. Cell Death Dis 4: e842, 2013.

33. Yang M, Yuan F, Li P, Chen Z, Chen A, Li S and Hu C: Interferon regulatory factor 4 binding protein is a novel p53 target gene and suppresses cisplatin-induced apoptosis of breast cancer cells. Mol Cancer 11: 54, 2012. 